\title{
Incipient CDW Order in the Pseudo-Gap Phase of the Cuprates
}

\author{
(Dated: October 28, 2012)
}

a) J. Chang et al, "Direct observation of competition between superconductivity and charge density wave order in $\mathrm{YBa}_{2} \mathrm{Cu}_{3} \mathrm{O}_{y}$ " arXiv:1206.4333. (Nat. Phys. published online 10/14/2012.)

b) G. Ghiringhelli et al, "Long-range incommensurate charge fluctuations in (Y,Nd) $\mathrm{Ba}_{2} \mathrm{Cu}_{3} \mathrm{O}_{6+x}$," Science 337, 821 (2012).

\section{Recommended with a commentary by Steven Kivelson, Stanford University}

One of the most intensely debated issues in the field of highly correlated electronic materials is the nature of the famous "pseudo-gap" regime in the phase diagram of the cuprate high temperature superconductors. While the existence of such a regime was originally inferred from the anomalous temperature dependence of the spin response (measured by NMR) and the resistivity, it has since been studied, with ever increasing precision and finesse, with just about every probe available to solid state physics. This regime is extremely interesting in its own right, as it embodies, in a sense, the most highly correlated (i.e. non-simply metallic) behavior in the phase diagram, and because at least over much of the phase diagram, it is the "normal" state out of which high temperature superconductivity emerges. Interpretations, typically which focus on one or another subset of the observed phenomena, range from identifying it with a broken symmetry phase with a difficult to observe order parameter, to associating it with fluctuating order associated with one or another proximate ordered phase, to being a signature of an actual, or approximate new "non-Fermi liquid" phase of matter.

In the last few months, two independent X-ray studies[a,b] on high quality underdoped crystals of the cuprate high temperature superconductor, YBCO, have detected pronounced peaks in the structure factor corresponding to substantially correlated charge-density-wave (CDW) fluctuations which emerge below a temperature, $T_{C D W} \approx 140 K$, which is lower than, but of order the conventionally defined pseudo-gap temperature, $T^{\star} \approx 250 \mathrm{~K}$, at the same level of doping. (Similar results have also been reported on thin films in [b], and further results on crystals in [1].) YBCO has a crystal structure consisting of stacked $\mathrm{Cu}-\mathrm{O}$ bilayers, and is strongly (electronically) orthorhombic due to the presence of $\mathrm{Cu}-\mathrm{O}$ chain layers. The new peaks in the X-ray structure factor are centered at the scattering vectors $\vec{Q}_{1}=\left(q_{1}, 0,1 / 2\right)$ and $\vec{Q}_{2}=\left(0, q_{2}, 1 / 2\right)$ with $q_{1} \approx q_{2} \approx 0.31$. The correlation length of the CDW order, obtained from the width of the superlattice peaks, is temperature dependent, but grows to a maximum value corresponding to a correlation length of roughly $\xi_{a b} \approx 100 \AA \sim 25 a$ lattice constants in plane, and $\xi_{c} \approx 1$ unit cell in the interplane direction. The value of $q_{1}$ implies that, in plane, the density wave order is incommensurate, with a period close to three unit cells (which would correspond to $q_{1}=1 / 3$ ). The " $1 / 2$ " in the ordering vector implies a tendency for neighbor- ing bilayers to have the CDW order shifted by phase $\pi$ relative to each other - if there were true long-range order, it would correspond to a new unit cell with $4 \mathrm{Cu}-\mathrm{O}$ planes. From the ratio of intensities of the new peaks and the Bragg peaks, Chang et al[a] estimate that the corresponding lattice displacements, $u$, in units of the lattice constant $a$, are of order $(u / a) \sim 10^{-3}$ or $U \sim 0.003 \AA$.

The fact that this fluctuating CDW order is strongly coupled to, and competes, at some level, with superconductivity is demonstrated by the non-monontonic temperature dependence of the scattering intensity and the correlation length; both grow with decreasing temperature for $T_{C D W}>T>T_{c}$, then drop with further decrease of temperature upon entering the superconducting state for $T<T_{c} \approx 60 \mathrm{~K}$. This competition is further corroborated by the high magnetic field studies of Chang et al[a], in which it is shown that application of magnetic fields up to $17 \mathrm{~T}$ perpendicular to the planes has no detectable effect on the CDW correlations for $T>T_{c}$, but produces a large magnetic field induced enhancement of both the intensity and the correlation length of the peak in the structure factor when $T<T_{c}$. Presumably, this low temperature enhancement of the CDW order is an indirect consequence of the magnetic field induced suppression of the superconducting order.

There are several aspects of the results that are worth highlighting. Firstly, given the strong electronic anisotropy of the materials, the fact that the peaks at $\vec{Q}_{1}$ and $\vec{Q}_{2}$ (which lie, respectively, along the orthorhombic $\mathrm{a}$ and $\mathrm{b}$ axes) are so similar in intensity, incommensurability, and correlation length is somewhat surprising. Somehow, the ordering in the $\mathrm{Cu}-\mathrm{O}$ planes seems impervious to the presence of the chains. Secondly, it is notable that the correlation length grows to be so substantial in the in-plane direction, but remains extremely short in the interplane direction. In the absence of frustration, one would expect the effective coupling between the CDW order parameter in neighboring planes to be enhanced by a factor of $\left(\xi_{a b}\right)^{2}$ over its bare value as inplane correlations develop.

Somewhat unaccountably, considering how extensively this material has been studied, this is the first report of direct evidence of CDW order in YBCO in this range of temperatures and magnetic fields. There have, however, been other experiments that are indicative of various forms of symmetry breaking in underdoped YBCO, with which the present results should be compared. 
Within the last year NMR studies[2] have found clear evidence of fully static CDW order at low temperatures and high magnetic fields (to suppress superconductivity) in Ortho-VIII crystals of YBCO (the same as in the Xray studies) and slightly more underdoped Ortho-II crystals. (The CDW order gives rise to a distinct quadrapolar splitting of the NMR line in the charge ordered state, which, in turn, gives a local measurement of the charge distribution surrounding different planar $\mathrm{Cu}$ sites.) However, there are several distinct differences between the charge order observed in NMR and the newly observed fluctuating CDW order in the X-ray experiments. Firstly, at least in the Ortho-II crystal, the CDW order inferred from the NMR is commensurate and has a single ordering vector, $\vec{Q}_{N M R}=(q, p, L)$ where $q=1 / 4, p=0,1 / 2$, or $1 / 4$ and $L$ is unknown. Neither the commensurate value of $q$, nor the unidirectional character of the inferred order are consistent with the incipient order inferred from X-rays. Moreover, the NMR-inferred order appears to onset at a sharp critical temperature (or critical magnetic field), implying the existence of a thermodynamic phase transition, while the fluctuating CDW order appears to onset at a crossover. It is possible that these differences are sufficiently strong that they indicate that the two CDW ordering tendencies seen in the same material may be entirely unrelated. For instance, it has been suggested that the fluctuational order may be associated with Fermi surface nesting, while the low temperature order is related to the stripe order seen in other cuprates. This is not the sort of unifying simplicity we hope for, but it cannot presently be ruled out.

Three other pieces of evidence of thermodynamic phase transitions in YBCO for the same range of doping should also be mentioned: 1) Spin-polarized neutron scattering[3] studies have yielded evidence of a transition to a (time-reversal symmetry breaking) intra-unit-cell antiferromagnetic state below a critical temperature that appears to correspond much more accurately to $T^{\star}$ than to $T_{C D W}$. It is presently uncertain what relation there is between any such translation symmetry preserving order and the CDW order which is our focus for now. 2) High resolution optical measurements[4] revealed, some time ago, the existence of a sharp onset temperature, $T_{K e r r}$, such that for $T<T_{K e r r}$, the Kerr angle, $\theta_{K e r r}$, measured in vertical incidence reflectivity, is non-zero. Comparing data for the same range of doping reveals that $T_{C D W} \approx T_{\text {Kerr }}$, suggesting that whatever transition is responsible for the Kerr onset is likewise associated with the growth of the CDW correlations. 3) Ultrasound measurements[5] have revealed two distinct features - an apparently non-analytic change in the sound velocity at a temperature which is identified as $T^{\star}$, and a peak in the ultrasonic attenuation rate at a temperature corresponding to $T_{C D W}$. This may support the notion that there are at least two distinct transitions.

There has been an overwhelming variety of other experiments which have explored the pseudo-gap onset in YBCO - too many to list here. A particular result [6] which may warrant special mention is the identification of a characteristic temperature, $T_{H}$, at which the Hall resistance begins to deviate downwards from its high temperature trend. (At still lower temperatures, for the relevant range of doping and if superconductivity is suppressed by the application of a high magnetic field, the Hall resistance eventually changes sign and is negative at the lowest temperatures.) $T_{H}$ has been speculatively identified as the temperature at which incipient density wave order (of unspecified character) begins to lead to a reconstruction of the Fermi surface. It is notable that $T_{H}$ as a function of doping concentration matches quite accurately to the previously determined values of $T_{K e r r}$, and hence in the range of doping in which the X-ray peak occurs, it also corresponds well to $T_{C D W}$. The importance of this observation is that it links the onset of CDW correlations with changes in the electronic structure, implying that the changes which occur associated with the onset of CDW correlations are significant.

One of the other notable advances in the field of the last few years is the observation of clear, clean quantum oscillations at low temperatures and high magnetic fields (up to 100T!). In addition to the zeroth order implication that there exist well defined electronic quasiparticles in the $T=0$ "normal" (resistive) state when superconductivity is suppressed, the fact that the inferred Fermi surface is electron-like and encloses only a small portion of the Brillouin zone implies that it has been substantially reconstructed relative to the underlying "bare" Fermi surface, which is known to be large and hole-like. Many proposals have been put forward implicating various forms of density wave order as the origin of this reconstruction, and it is therefore attractive to associate the newly identified fluctuating CDW order with this Fermi surface reconstruction. On the other hand: 1) The "fluctuating" order must become static in order to give rise to quantum oscillations; the order detected by NMR is certainly static, so this observation emphasizes the need to reconcile the differences between the two sets of results. 2) Without perfect nesting, it is hard to imagine that density wave order alone, especially the relatively weak order seen in X-rays, can eliminate large portions of the Fermi surface, away from well-nested "hot-spots," as appears to be necessary to explain the low $T$ experiments.

Finally, it is worth comparing the present structure with what is known about CDW ordering in other cuprates. Unambiguous evidence of unidirectional charge density wave order ("charge stripes") has been obtained in a subset of crystals within the so-called 214 family of cuprates. For instance[7], in $\mathrm{La}_{2-x} \mathrm{Ba}_{x} \mathrm{CuO}_{4}$ with $x=1 / 8$, sharp peaks in the structure factor observed both with x-rays and neutrons occur at $\vec{Q}_{1}=\left(q_{1}, 0,1 / 2\right)$ and $\vec{Q}_{2}=\left(0, q_{2}, 1 / 2\right)$ with $q_{1}=q_{2}=0.241 \neq 0.25$. These peaks are much sharper than those observed in YBCO, with an in-plane correlation length which can approach $10^{2}$ lattice constants, although the correlation length in the inter-plane direction is, again, of order the size of a 
unit cell. It is known that these peaks arise from a pattern of stripes which rotate by $\pi / 2$ in going from plane to plane, so the peaks at $\vec{Q}_{1}$ and $\vec{Q}_{2}$ arise, respectively, from the alternating planes that have stripes running parallel to the $\mathrm{y}$ axis and $\mathrm{x}$ axis. Moreover, presumably as a consequence of the longer correlation length, the onset of CDW ordering occurs below a sharply defined critical temperature. It is notable that the Kerr effect, measured $[8]$ in the same material, again exhibits a sharp onset with $T_{K e r r}=T_{C D W}$. It is also notable that incommensurate magnetic order ("spin stripe order") onsets at a second, well defined transition temperature, $T_{S D W}$, which is $10 \mathrm{~K}$ lower than $T_{C D W}$.

Moving from bulk to surface probes, and the BSCCO family of cuprate superconductors, a large number of STM studies (especially from the Davis, Kapitulnik, and Yazdani groups) have found evidence of a prominent tendency toward CDW formation, and have clearly associated this tendency with spectroscopic features associated with the pseudo-gap. Indeed, the CDW correlation length found in these studies is typically in the same ball-park of 20 lattice constants, as those observed in the X-ray studies of YBCO, although the ordering vectors, $\vec{Q}_{1}=(q, 0, L)$ and $\vec{Q}_{2}=(0, q, L)$, are typically in the range $q \sim 0.2$, which is even smaller than that associated with the stripe order in LBCO.

Clear evidence of incipient $\mathrm{CDW}$ order in $\mathrm{YBCO}$ is an important advance in the field. YBCO is generally thought to be the "cleanest" of the cuprate materials, and so ordering tendencies observed in this material are probably intrinsic. There is no doubt that correctly identifying the forms of order that occur in the relevant portion of the cuprate phase diagram is an important step toward unraveling their mysteries. None-the-less, there remain many obvious questions concerning the interpretation of these results: Is the incipient CDW order in YBCO a material specific property of this family of cuprates in a narrow range of doping, or is it more ubiquitous? Is it a close relative of the fully formed CDW order seen in the same materials (by NMR) at higher fields and low temperatures? Is it closely related to the well developed stripe order seen in the 214 family of cuprates and/or the short-range CDW correlations seen on the surface of BSCCO? On a more basic level, is the incipient CDW order always correlated with pseudo-gap formation? Is local CDW ordering the cause of the pseudo-gap or a derivative phenomenon that can, at times, arise once the essential pseudo-gap correlations have developed? Is there more to the interplay between CDW order and high temperature superconductivity than just "competing orders."

[1] A. J. Achkaret al, "Distinct Charge Orders in the Planes and Chains of Ortho-III-Ordered $\mathrm{YBa}_{2} \mathrm{Cu}_{3} \mathrm{O}_{6+\delta}$ Superconductors Identified by Resonant Elastic X-ray Scattering," Phys. Rev. Lett. 109 , 167001 (2012).

[2] T. Wu et al, "Magnetic-field-induced chargestripe order in the high temperature superconductor $\mathrm{YBa}_{2} \mathrm{Cu}_{3} \mathrm{O}_{y}$," Nature 477, 191 (2011).

[3] B. Fauque et al, "Magnetic order in the pseudogap phase of high- $T_{c}$ superconductors," Phys. Rev. Lett. 96, 197001 (2006)

[4] J. Xia et al, "Polar Kerr Effect Measurements of $\mathrm{YBa}_{2} \mathrm{Cu}_{3} \mathrm{O}_{6+x}$ : Evidence for Broken Symmetry Near the Pseudogap Temperature," Phys. Rev. Lett. 100, 127002 (2008).

[5] A. Shekhter et al, "Ultrasonic signatures at the superconducting and the pseudogap phase boundaries in YBCO cuprates," arXiv:1208.5810.

[6] N. Doiron-Leyraud, L. Taillefer, "Quantum critical point for stripe order: An organizing principle of cuprate superconductivity," Special issue of Physica C on "Stripes and electronic liquid crystals," Physica C 481, 161 (2012).

[7] S.B. Wilkins, M. P. M. Dean, J. Fink, M. Hcker, J. Geck, V. Soltwisch, E. Schierle, E. Weschke, G. Gu, S. Uchida, N. Ichikawa, J.M. Tranquada, J.P. Hill, "A Comparison of Stripe Modulations in $\mathrm{La}_{1.875} \mathrm{Ba}_{0.125} \mathrm{CuO}_{4}$ and $\mathrm{La}_{1.48} \mathrm{Nd}_{0.4} \mathrm{Sr}_{0.12} \mathrm{CuO}_{4}$, Phys. Rev. B 84, 195101 (2011).

[8] H. Karapetyan et al, "Magneto-optical signatures of a cascade of transitions in $\mathrm{La}_{2-x} \mathrm{Ba}_{x} \mathrm{CuO}_{4}$," Phys. Rev. Lett. 109, 147001 (2012). 